\title{
Educação em saúde: aspectos relevantes apontados por adolescentes
}

\section{Health education: significant features pointed out by teenagers}

\author{
Juliana Alvares Duarte Bonini CAMPOS \\ Doutoranda - Programa de Pós-Graduação em Odontologia - Área de Odontopediatria - Faculdade de Odon- \\ tologia de Araraquara - UNESP
}

Ângela Cristina Cilense ZUANON

Professora Assistente Doutora - Departamento de Clínica Infantil - Faculdade de Odontologia de Araraquara UNESP

\begin{abstract}
Resumo
A educação em saúde é uma importante estratégia no processo de formação de comportamentos que visem a promoção de saúde. O diagnóstico do conhecimento da maneira de como é vista a questão da saúde por cada indivíduo é fundamental para elaboração de programas educativos eficientes. O presente trabalho foi realizado em uma escola particular da cidade de Araraquara (SP) onde avaliou-se por meio de questionário a opinião de 244 adolescentes de 12 a 16 anos de idade quanto aos aspectos mais relevantes para a promoção de saúde. Os resultados demonstraram que apesar de $40 \%$ dos adolescentes afirmarem que a alimentação adequada é importante para a manutenção da saúde, apenas $8,4 \%$ destes sabiam o porque desta relação. Dentre os assuntos mais conversados entre os adolescentes estão a alimentação (17,5\%), a prática de esportes (12,3\%) e a AIDS (20,9\%). De acordo com os entrevistados, as informações para promoção de saúde deve partir dos profissionais da área $(62,1 \%)$. Conclui-se portanto que apesar de os adolescentes apresentarem informações pertinentes para a elaboração do conceito de saúde, estas devem ser trabalhadas para a tomada de uma postura adequada para a real promoção de saúde.
\end{abstract}

\section{UNITERMOS}

Educação, saúde; adolescentes; saúde, promoção

\section{INTRODUÇÃO}

A partir da década de setenta, a promoção de saúde têm sido associada à ação de educadores priorizando a responsabilidade e motivação de cada indivíduo para a mudança de comportamento $^{4}$.

O setor educacional é um aliado importante para a concretização de ações de promoção de saúde voltadas para o fortalecimento das capacidades dos indivíduos, para a tomada de decisões favoráveis à saúde e à comunidade (BRASIL ${ }^{2}$, 1996).

Entretanto, para se educar é necessário conhecer a realidade e o pensamento de cada indivíduo a ser incluído em um programa, para que se possa apresentar conceitos que tenham influencia sobre os mesmos.

Sabendo-se que a escola é o local onde os adolescentes passam grande parte de sua vida, atuan- do de maneira significativa na formação de opiniões e na construção de caráter, passa a ser um local de referência para a implementação de qualquer programa que vise a educação ${ }^{3}$.

Desta forma realizou-se um estudo com o intuito de verificar quais são os aspectos mais relevantes na educação em saúde apontados por um grupo de adolescentes de uma escola particular da cidade de Araraquara.

\section{Material e mÉtodos}

Para realização deste estudo, foi aplicado um questionário (Anexo 1), respondido por alunos matriculados de $5^{\circ}$ a $8^{\circ}$ série do ensino fundamental, de uma escola particular da cidade de Araraquara (SP). A amostra constituiu-se de 480 alunos, sendo que a participação era voluntária e os questionários identificados apenas pela série. 
Campos JADB, Zuanon ACC

EDUCAÇÃO EM SAÚDE: ASPECTOS RELEVANTES APONTADOS POR ADOLESCENTES

Antes da execução do trabalho obteve-se a aprovação junto à direção da escola e realizou-se instruções aos professores, os quais aplicaram o questionário, esclarecendo os alunos sobre os objetivos e a importância do estudo. O professor em seguida, saiu da sala para deixar que os alunos respondessem as questões sem nenhum tipo de constrangimento.
Em seguida, as respostas foram lidas e agrupadas de acordo com seu conteúdo. Logo após, procedeu-se a análise estatística descritiva através da distribuição de frequências relativa e absoluta.

\section{Resultados}

Os resultados estão representados na Tabela 1 e nas Figuras de 1 a 3.

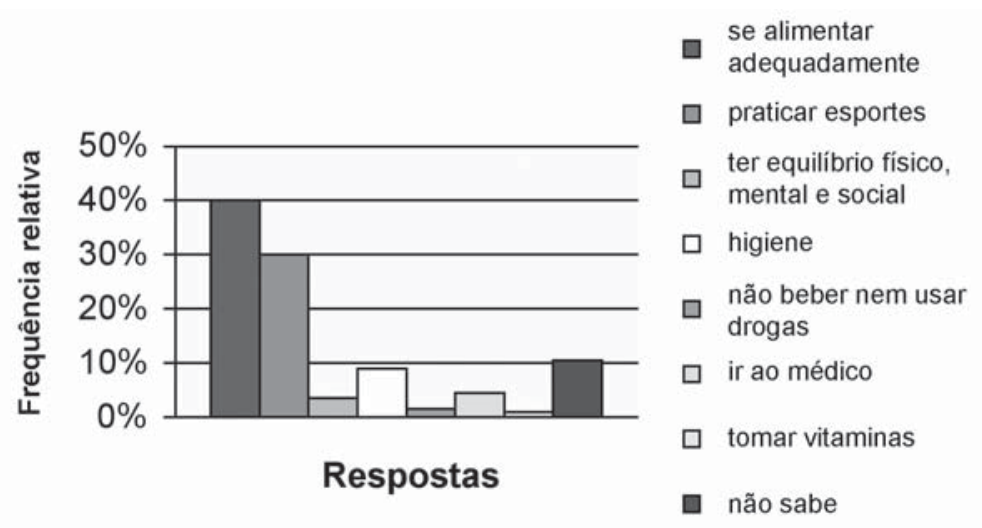

FIGURA 1 - Distribuição de frequências relativas do conhecimento dos adolescentes referentes ao que é preciso fazer para se ter saúde.

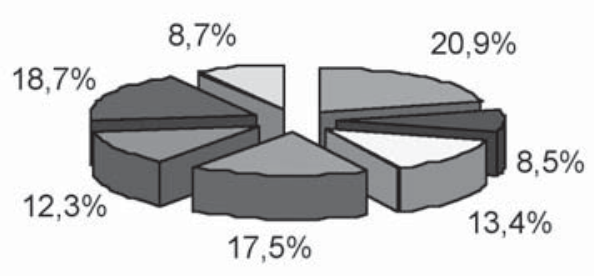

$\square$ AIDS

$\square$ gravidez

$\square$ Higiene bucal

$\square$ alimentação

$\square$ esportes

outros

$\square$ todos

FIGURA 2 - Distribuição de freqüências relativas dos assuntos relacionados à saúde, mais conversados entre os adolescentes. 
Campos JADB, Zuanon ACC

EDUCAÇÃO EM SAÚDE: ASPECTOS RELEVANTES APONTADOS POR ADOLESCENTES

Tabela 1 - Distribuição de frequências das respostas referentes à interferência da alimentação na saúde e o modo de ocorrência

\begin{tabular}{|c|c|c|}
\hline Perguntas/Respostas & $(\%)$ & (n) \\
\hline \multicolumn{3}{|c|}{ Você acha que, o que você come pode interferir na sua saúde? } \\
\hline Sim & 74 & 357 \\
\hline Não & 26 & 123 \\
\hline Total & 100 & 480 \\
\hline \multicolumn{3}{|l|}{ Em caso afirmativo, de que modo? } \\
\hline Pode provocar anemia & 1,4 & 5 \\
\hline Pode provocar doenças & 11,8 & 42 \\
\hline Pode engordar & 5,9 & 21 \\
\hline Pode dar problemas cardíacos & 19,9 & 71 \\
\hline Comer porcarias faz mal & 27,7 & 99 \\
\hline Para manter o equilíbrio do organismo & 8,4 & 30 \\
\hline Não sabe & 24,9 & 89 \\
\hline Total & 100 & 357 \\
\hline
\end{tabular}

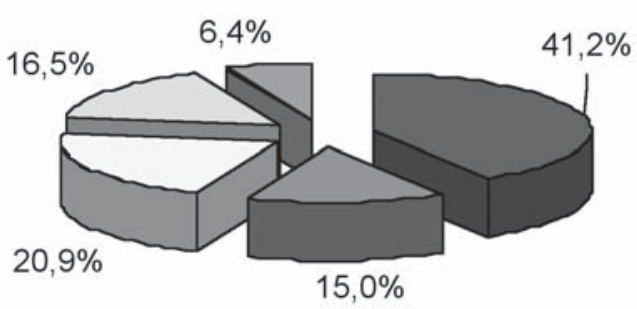

$\square$ médico

$\square$ professores

outros profissionais

de saúde

$\square$ todos

口 não sabe

FIGURA 3 - Distribuição de freqüências relativas referentes ao profissional responsável pela educação em saúde, segundo os adolescentes. 
Campos JADB, Zuanon ACC

EDUCAÇÃO EM SAÚDE: ASPECTOS RELEVANTES APONTADOS POR ADOLESCENTES

\section{Dıscussão}

A educação é considerada por Volschan \& Soares $^{11}$ (2002), um processo básico no trabalho de promoção de saúde. A elaboração deste estudo, esteve vinculada à idéia de Tamietti et al. ${ }^{9}$ (1998), de que para construção de conceitos, deve-se anteriormente realizar um diagnóstico da imagem feita por cada indivíduo em relação ao tema proposto. Desta maneira, deve-se elaborar trabalhos preliminares antes da implementação de qualquer programa de educação.

A escolha de uma escola particular deveu-se ao fato de que seus alunos são diferenciados e provavelmente tem acesso aos serviços essenciais. Portanto, provavelmente já tiveram a oportunidade de conversar com profissionais capacitados para incorporação de conceitos sobre saúde.

A aplicação do questionário buscou levantar os aspectos mais relevantes apontados pelos adolescentes para construção do conceito de saúde. Pela observação da Figura 1 pode-se notar que 30\% apontou para necessidade de fazer esportes como condição para se ter saúde. Pôde-se observar porém, que os adolescentes referiram-se às atividades físicas apenas pensando na manutenção estética do corpo, não considerando os benefícios fisiológicos, sociais e emocionais que estão relacionados esta prática.

Este fato, demonstra que a indicação de esportes pelos adolescentes como condicionante da saúde é apenas uma informação isolada e não um conceito bem estabelecido, concordando com Vente et al. ${ }^{10}$ (2001) quando diferencia informação de educação.

Apesar de $74 \%$ dos adolescentes afirmarem que a alimentação pode interferir na saúde, apenas $8,4 \%$ demostrou saber da relação existente entre o processo de alimentação e a homeostase orgânica (Tabela 1).

Este tipo de resultado foi também encontrado por Vente et al. ${ }^{10}$ (2001) que demonstraram que apesar dos adolescentes possuírem grande quantidade de informações sobre alimentação e sobre saúde não existe um conceito estabelecido. Este fato demonstra que na maioria das vezes, a educação não é trabalhada e reforçada como deveria para sua assimilação e concretização do processo de promoção de saúde.

Lima et al. ${ }^{6}(2000)$ ressaltam ainda que para a incorporação e elaboração de conceitos, a educação deve receber uma abordagem humanística, sendo capaz de promover crescimento pessoal, inter- pessoal e grupal. Com relação à orientação alimentar, Bissoli \& Lanzillotti ${ }^{1}$ acrescentam ainda, que o processo de educação deve seguir etapas de intervenção caracterizadas por, diagnóstico das informações apresentadas pelo grupo, medidas antropométricas e posterior elaboração de um programa. Lembra ainda que não se deve esquecer das peculiaridades do grupo assistido com o envolvimento real de toda equipe, desde a direção da escola até os professores e profissionais de saúde.

A busca de responsabilidade para a elaboração de um programa deveria considerar a interdisciplinariedade perguntando o que se quer ensinar, para quem e com que objetivo, para só então identificar quais serão os agentes de interação.

No Figura 2, nota-se mais uma vez, a alimentação e os esportes permeando o diálogo dos adolescentes quando a questão é saúde $(38,4 \%)$. Entretanto como discutido acima, seus conceitos são superficiais e não representam o envolvimento real destes condicionantes na vida destes indivíduos. Pode-se verificar também que 20,9\% dos adolescentes conversam sobre AIDS o que pode ser justificado pela grande preocupação despertada nos governantes, na mídia e de um modo geral na população (Figura 2).

Nobrega et al. ${ }^{7}$ (1991) verificaram que apesar da preocupação sobre a AIDS apresentadas pelos adolescentes, não há consciência suficiente para um comportamento adequado para a prevenção da doença. Assim, Cunha et al..$^{5}$ (1998) elaborou um programa educativo para adolescentes em João Pessoa $(\mathrm{PB})$ e constatou que houve melhora no conhecimento sobre a doença, justificando assim a continuidade do trabalho e a recomendação de ampliação do mesmo entre as escolas do município.

Frente à variedade de assuntos apontados pelos adolescentes quando da educação em saúde verificase que faz-se necessária uma atuação interdisciplinar.

Segundo Rabuske ${ }^{8}$ (2001) a prática interdisciplinar é uma alternativa metodológica voltada para a superação das dificuldades decorrentes da fragmentação do conhecimento, constituindo-se de um esforço coordenado entre várias disciplinas científicas e da articulação entre teoria e prática. Assim, diversas categorias de profissionais necessitariam rever seu trabalho, através de um espírito crítico, abertos à cooperação, ao intercâmbio, ao questionamento e à discussão num processo permanente.

Desta forma, entende-se que o processo de educação em saúde deve estar incluído dentro das práticas interdisciplinares tanto dos professores quanto 
Campos JADB, Zuanon ACC

EDUCAÇÃO EM SAÚDE: ASPECTOS RELEVANTES APONTADOS POR ADOLESCENTES

dos profissionais de saúde, não devendo portanto haver a atribuição única de responsabilidade. Este tipo de conduta porém, foi observada em apenas $16,5 \%$ dos entrevistados, salientando assim a visão fragmentada de educação vigente cotidianamente, onde a orientação em saúde é obrigação exclusiva do médico $(41,2 \%)$ (Figura 3 ) .

\section{Conclusão}

1. Apesar dos adolescentes terem apresentado informações pertinentes para a elaboração do conceito de saúde, estas devem ser trabalha- das para a tomada de uma postura mais adequada para a real promoção de saúde.

2. A educação em saúde deve ser realizada após o diagnóstico dos aspectos mais relevantes apontados pelo grupo a ser beneficiado com o intuito de propiciar uma elaboração correta de conceitos.

3. Deve-se contar com um envolvimento multiprofissional que possua como objetivo único o favorecimento de um crescimento pessoal, social e emocional de cada indivíduo para a elaboração de programas educacionais.

\begin{abstract}
Health education is an important strategy in the process of behavior formation aimed at health promotion. Diagnosis of knowledge about the way each individual perceives the health matters is of paramount importance in working out efficient educational programs. The conducted study was performed in a private school in the city of Araraquara (Brazil), in which the opinions of 244 teenagers, aged 12 to 16, were availed by means of a questionnaire proposed to them. This questionnaire concerned the most significant features of health promotion. The obtained results reveal that $40 \%$ of the teenagers stated that correct eating habits are important for maintaining a good health, but only $8.4 \%$ of them knew the reasons for this relationship. Among the subjects most talked-about by these teenagers figure food (17.5\%), sports practice (12.3\%) and AIDS (20.9\%). According to the interviewed teenagers, specialists from the health area should provide information about promotion of good health practices. It may be concluded that even though the teenagers have adequate information for acquiring health concepts, this information should be properly ordered for an adequate attitude towards a real health promotion.
\end{abstract}

\title{
UNITERMS
}

Education, health; teenagers; health, promotion; adolescent health

\section{REFERÊNCIAS}

1. Bissoli MC, Lanzillotti HS Educação nutricional como forma de intervenção: avaliação de uma proposta para pré-escolares. Rev Nutr PUCCAMP 1997 jul./dez.; 10(2):107-13.

2. Brasil. Ministério da Saúde. A promoção da saúde no contexto escolar. Rev saúde Pública 2002 ago; 36(2):533-5.

3. Burghardt JA, Devaney BL, Gordon AR. The school nutrition dietary assessment study: summary and discussion. Am J Clin Nutr 1995 Jan.; 61(1):252-7.

4. Chor D, Faerstein E. Um enfoque epidemiológico de promoção de saúde: as idéias de Geoffrey Rose. Cad Saúde Pública 2000 jan./mar.; 16(1):241-4.
5. Cunha MMLC, Soares MJGO, Novo SMJ, Costa SPR. Avaliação de uma proposta educativa sobre AIDS com adolescentes de escola pública de João Pessoa - PB. Ver Bras Ciênc Saúde 1998 dez.; 2(1/3):27-32.

6. Lima RT, Barros JC, Melo MRA, Sousa MG. Educação em saúde e nutrição em João Pesooa, Paraíba. Rev Nutr PUCCAMP 2000 jan./abr.; 13(1):29-36.

7. Nobrega MM, Sá LD, Nobrega MML. Conhecimento e opiniões dos adolescentessobre AIDS. Cad Enfermagem Ciênc e Cult 1991; 5(2):64-81.

8. Rabuske CHSA. Interdisciplinaridade como condição na intervenção psicopedagógica. Reflexão e Ação 2001 jan./jun.; 9(1):8591. 
Campos JADB, Zuanon ACC

EDUCAÇÃO EM SAÚDE: ASPECTOS RELEVANTES APONTADOS POR ADOLESCENTES

9. Tamietti MB, Castilho LS, Paixão HH. Educação em saúde bucal para adolescentes: inadequação de uma metodologia tradicional. Arq Odontol 1998 jan./jun.; 34(1):33-45.

10. Vente W, Post GB, Twisk J, Kemper HCG, Mechelen W. Effects of health measurements and health information in youth and yong adulthood in dietary intake-20y study results from the Amsterdam growth and health longitudinal study. Eur J Clin Nutr 2001 Oct.; 55(10):819-23.

11. Volschan BCG, Soares EL. Educação em saúde. Rev ABOPREV 2002; 5(2):27-32.

ANEXO 1: Questionário aplicado aos alunos de $5^{\circ}$ a $8^{\circ}$ série de ensino fundamental. Araraquara. 2002.

1 - O que é preciso fazer para se ter saúde?

2 - Você acha que, o que você come pode interferir na sua saúde?

( ) $\operatorname{sim}($ ) não

3 - Em caso afirmativo, de que modo?

4 - Em relação à saúde, o que vocês e seus amigos conversam?

5 - Na sua opinião, a saúde deveria ser abordada por que tipo de profissional?

Recebido em: 02/10/03

Aprovado em: 12/12/03

Juliana Alvares Duarte Bonini CAMPOS Avenida Brasil, $n^{\circ} 740$, apt $^{\circ} 82$

Centro, Araraquara (SP)

Cep: 14801-050

acampos@foar.unesp.br 